\title{
El tratamiento de la metáfora en la traducción de Historias de Tata Mundo ${ }^{1}$
}

\section{(The Treatment of the Metaphor in the Translation of Historias de Tata Mundo)}

Jacqueline Araya Ríos ${ }^{2}$

Universidad Nacional, Costa Rica

\section{resumen}

Fabián Dobles plantea mediante hechos característicos y regionalismos una imagen del campesino nacional de mediados de siglo xx en su obra Historias de Tata Mundo. Joan Henry lo traduce al inglés en 1998 y lo titula The Stories of Tata Mundo, transportando al campesino costarricense a la escena de habla inglesa. Se analizan las decisiones de la traductora sobre el tratamiento de las metáforas, teniendo en cuenta el lenguaje figurado como una herramienta empleada por el autor para caracterizar la vida rural del campesinado costarricense.

\begin{abstract}
Using traditional activities and regionalisms, Fabián Dobles reconstructed the image of the Costa Rican country folk of the mid-twentieth century in his literary work Historias de Tata Mundo. Joan Henry translated it in 1998, with the title The Stories of Tata Mundo, transporting the local peasant to the English-speaking scenario. The translator's decisions are analyzed
\end{abstract}

1 Recibido: 23 de marzo de 2014; 26 de julio de 2014. Versión revisada de la ponencia leída en el marco del III Congreso Internacional de Lenguas Modernas, Costa Rica 2012, «Convergencia de Lenguas y Culturas», llevado a cabo entre el 10 y el 13 de diciembre de 2012, en la Universidad de Costa Rica, San José de Costa Rica.

2 Departamento de Idiomas Extranjeros, Sede Regional Brunca. Correo electrónico: jacqueline. araya.rios@una.cr 
here regarding the translation of metaphors, taking into account figurative speech as a tool used by the author to characterize and depict the rural life of Costa Rican country folk.

Palabras clave: narrativa costarricense, Fabián Dobles, lenguaje figurado Keywords: Costa Rican narrative, Fabián Dobles, figurative speech

The stories of Tata Mundo include some of the most attractive stories I have ever read. Through them I have learnt about the people and country of Costa Rica and admired greatly the qualities of Fabián Dobles as a writer and human being. Above all, I have been made to feel the strong, inextricable bond between the writer and his people.

Joan Henry

\section{Introducción}

A eso de las once, íbamos los dos por la carretera que se deslizaba hacia el cercano barrio de San Jerónimo, abriéndonos vereda por entre la oscuridad con la vieja linterna del cura. Todo dormía: los cafetales, los potreros, las vacas que adivinábamos echadas a la vera del camino. Sólo algún perro bostezaba a ladridos sintiéndonos pasar y los cuyeos salpimentaban aquí y allá el ancho silencio nocturno. A mí la espalda me brincaba como un animal, del miedo. Pero a esa edad uno se las da de hombrecito, y le echaba siete nudos a mi frío para que las quijadas no me temblequearan más de la cuenta. Sin embargo, ustedes adivinarán que cuando entramos al cementerio lo menos que hacían mis dientes era sonar como matracas ${ }^{3}$.

Cuando Nida, Dagut y Vinay y Darbelnet (cito por Samaniego ${ }^{4}$ ) hablan de la intraducibilidad de la metáfora, han de haber estado ante un texto con la peculiaridad y originalidad que caracteriza el extracto anterior. ¡Qué valentía de la persona que se tira al ruedo y decide

3 Fabián Dobles, Historias de Tata Mundo (San José, Costa Rica: Editorial Costa Rica, 1978) 8. Las referencias se tomarán de esta edición y de ahora en adelante se indicará las páginas entre paréntesis después de la cita.

4 Eva Samaniego, La traducción de la metáfora (Valladolid: Servicio de Publicaciones de la Universidad de Valladolid, 1996) 69. 
emprender dicha empresa! Pues los méritos se los debe llevar Joan Henry, profesora de lenguas y traductora quien se declara admiradora de las dotes humanitarias de Fabián Dobles, más conocido por sus novelas El sitio de las abras y Ese que llaman pueblo. Estas, junto con Aguas turbias, han sido traducidas a varios idiomas (inglés, ruso y alemán), y han recibido reconocimiento en elámbito latinoamericano, al punto que su novela Ese que llaman pueblo ha sido comparada con The Grapes of Wrath, de Steinbeck.

Historias de Tata Mundo es una colección de cuentos de pueblo que narra las vivencias de un personaje del terruño costarricense, el «cuentacuentos». Tatamundo 'se las sabe de todas... todas' como diría el mismísimo Dobles. El mayor atractivo de la obra es ese uso tan peculiar del idioma que caracteriza a la zona rural de nuestro país; además, la simpatía y la ingenuidad que se refleja en los hechos y en los modismos con los que Dobles elabora la condición del campesino nacional de mediados de siglo xx. Es ese personaje costarricense que la traductora Joan Henry transporta a la escena de habla inglesa atraída por la gran destreza de Dobles como escritor, su comprensión del ser humano, el amor por su país, la identificación con su gente, que le permitieron darse a conocer. Más allá del sentimiento que podría despertar en una traductora que comparte con Dobles el de ser hija de esta patria, mi propósito es analizar la versión inglesa de la obra Historias de Tata Mundo, en cuanto al tratamiento de las metáforas, teniendo en cuentaque ellenguaje figurado es un recurso empleado para caracterizar y resaltar la vida rural del sector campesino costarricense.

\section{El lenguaje figurado}

Parto de lo general a lo específico, sin perder de vista que el propósito que nos embarga es resaltar y honrar la de Fabián Dobles, y cómo la traducción de su obra se convierte en la ventana a través de la cual el lector internacional, en inglés, lengua que comunica a 
las naciones, visualiza y conceptualiza al escritor costarricense, su pueblo, sus costumbres, su historia, en fin su realidad.

Según M. H. Abrams, el lenguaje figurado se ha clasificado en dos grandes grupos:

1) 'figures of thought,' or tropes (meaning 'turns,' 'conversions'), in which words are used in a way that effects a decided change or extension in their standard meaning; and (2) 'figures of speech,' or 'rhetorical figures,' in which departure from standard usage is not, primarily, in the meaning but in the order and rhetorical effect of the words 5 .

Entre las figuras están el símil, la hipérbole, la personificación, la metonimia y la sinécdoque. No obstante, la que más atrae al traductor es la metáfora; para algunos, la diferenciación entre los campos semánticos de la metáfora y la sinonimia y la sinécdoque no es muy clara.

\section{La metáfora}

Según Abrams, «In a metaphor a word which in standard (or literal) usage denotes one kind of thing, quality, or action is applied to another, in the form of a statement of identity instead of comparison» ${ }^{6}$. Por medio de una metáfora de Spender, realiza un análisis de las siguientesestrofas: «Eye, gazelle, delicate wanderer/Drinkerofhorizon's fluid line». Para el análisis de la figura, emplea el método propuesto por I. A. Richards que se compone de dos elementos, el tenor «for the subject to which the metaphoric word is applied (eye)», y el vehículo «for the metaphoric word itself (gazelle, wanderer y drinker). Peter Newmark ${ }^{7}$ nos habla de «un área semántica común entre dos —más o menos- cosas semejantes: la imagen y el objeto» y que constituye según el autor un proceso y no una función. Agrega:

5 M. H. Abrams, A Glossary of Literary Terms. 3a ed. (Nueva York: Holt, Rinehart and Winston, 1971) 60 .

6 Abrams, 61.

7 Peter Newmark, Manual de traducción (Madrid: Cátedra, 1992) 148. 
Uno de los problemas que presenta la traducción y comprensión de una metáfora original, de una metáfora adaptada o, en menor grado, de una estándar es decidir cuánto espacio hay que asignar al área de líneas entrecruzadas del sentido y determinar, luego, si ésta es: a) positiva o negativa; b) connotativa o denotativa ${ }^{8}$.

Este es un aspecto que se desarrolla más adelante, al analizar la traducción de ciertas figuras literarias en Historias de Tata Mundo. Entre tanto, observemos brevemente qué distingue a la metáfora de la metonimia y la sinécdoque.

\section{Metonimia y sinécdoque}

La metonimia ( Greek for 'a change of name» ${ }^{9}$ ) es un procedimiento estilístico que consiste en designar una cosa con el nombre de otra con la cual guarda una relación cercana: el laurel por la gloria, la canas por la vejez, la corona o el cetro por el rey o el reino. La sinécdoque ( Greek for 'taking together» ${ }^{10}$ ) es un procedimiento que consiste en «tomar una parte por el todo (pagar tanto por cabeza) o el todo por una parte (comprar un visón), o la materia de una cosa por la cosa misma, como el pan por toda clase de alimento» ${ }^{11}$. En inglés son frecuentes ejemplos como the hospital called, the embassy lobbied to hasten their deportation y you have to include more greens in your diet. En español son habituales expresiones como «está metido en un problema de faldas» 0 «no le gusta el mar» (cuando en realidad no es sólo el mar, sino la playa, el calor, los mosquitos, etc.). Puede observarse con estos ejemplos que en la práctica la línea que separa estas figuras no suele ser tan clara y definida. Si retomamos el ejemplo de Abrams, «eye» representa la capacidad de ver, observar, vigilar y hasta controlar, como en la frase «el ojo de la justicia», que constituye una sinécdoque.

8 Newmark, 148.

9 Abrams, 62.

10 Abrams, 62.

11 Ramón García-Pelayo y Gross, Larousse Diccionario usual (México: Ediciones Larousse, 1985) 617. 
A la identificación de la figura literaria, se le une el del aspecto idiosincrático del registro empleado por Fabián Dobles para moldear sus personajes con el perfil de lo que constituye el campesino costarricense de mediados del siglo pasado. Hay casos en que para el lector nacional contemporáneo, realizar una interpretación válida de algunos de los giros empleados no es tarea fácil. Con el fin de ilustrar observemos las frases: «no ser tan alejandro en puño» (10-11), «lo que en plata blanca averiguó» (50), «ya tenía alunados los lomos de tanta albarda» (51) y «lo tenía tan apersogado al bramadero» (58), las cuales requieren de un nivel de examen contextual significativo para su deducción y comprensión. The Craft of Translation explica que parte del conocimiento profundo que un hablante, y agregamos en especial un escritor, debe tener es:

A thoroughgoing familiarity with local expression and idiom... In many cases this closeness to regional expression makes translation difficult, sometimes impossible when it comes to preserving the flavor of the original ${ }^{12}$.

Este 'sabor' a terruño 'tico' es el eje que unifica los hechos y los personajes y da coherencia a sus actitudes, reflexiones y acciones. A la vez, ese folclore hecho personaje en Tata Mundo se convierte, junto con algunos otros personajes secundarios que el lector también puede «escuchar», lo que solía ser la escena campestre costarricense, con sus creencias, valores y condiciones que Dobles rescata y preserva para la posteridad en su obra. El traductor está consciente de estos factores que constituyen el acervo cultural y la riqueza del texto y debe conocer el sitio que ocupa dentro de nuestro sistema literario, para partir de ahí en la toma de decisiones que lo llevarán a la deconstrucción y posterior reconstrucción de otro texto de similares características, pero que ocupará otro sitio y otra función dentro del sistema meta;

12 John Biguenet y Rainer Schulte, eds. The Craft of Translation (Chicago: University of Chicago, 1989) 10. 
esta vez como vitrina de una cultura que le es extraña al lector y que, no obstante, le puede ser familiar y hasta simpática y ocurrente si el traductor ha logrado su cometido.

Debe considerarse que más allá de la destreza lingüística y el conocimiento que el autor revela sobre la cultura rural del terruño costarricense, el lenguaje figurado es el vehículo con el que Dobles condensa y expone la sabiduría de pueblo, sus credos, anhelos y temores, en fin su identidad labriega, la cual debe ser trasvasada a la audiencia meta por medio de otras metáforas que recreen ese sentido y esa identidad rústica y sencilla de nuestros campesinos. Entendemos que la empresa no es nada simple, que el reto se convierte en algunas ocasiones en un verdadero malabar, si recordamos las expresiones que anotamos anteriormente y que como hablantes de la lengua fuente nos son particularmente difíciles de descifrar. Como dice Lefevere: «Language is the expression of a culture, many of the words in a language are inextricably bound up with that culture and therefore very hard to transfer in their totality to another language $»^{13}$.

\section{El tratamiento de la metáfora en Stories of Tata Mundo ${ }^{14}$}

En el análisis realizado se tomaron en cuenta ciento treinta metáforas, de las cuales treinta fueron extraídas del cuento «El responso» y cien de «La bruja». Se identificaron varias estrategias empleadas por la traductora en su versión al inglés; entre ellas, omisión de la figura, traducción literal, neutralización o naturalización de la metáfora, equivalencia, simplificación y equivalencia con pérdida de la fuerza semántica. A pesar de que en promedio el trasvase de la figura revela tino por parte de Henry, también hubo desaciertos, especialmente en

13 André Lefevere, Translating Literature (Nueva York: The Modern Language Association of America, 1992) 17.

14 Fabián Dobles, The Stories of Tata Mundo. Trad. Joan Henry (San José, Costa Rica: Editorial de la Universidad de Costa Rica, 1998). Las referencias se tomarán de esta edición y se indicarán las páginas entre paréntesis después de la cita. 
aquellas locuciones que fueron malinterpretadas debido a la dificultad semántica, léxica o sintáctica de las construcciones.

Nida y Taber proponen tres maneras de transferir el lenguaje figurado:

a) cambios de uso figurado por uso no figurado, como 'dominar la puerta' por 'dominar la ciudad', 'mi carne' por 'mi raza', 'gustar la muerte' por 'morir'; b) cambios de un tipo de expresión figurada por otro, como 'corazón' por 'hígado' (cosa que sucede en numerosas lenguas africanas), 'alabar al Señor con la lengua' por 'alabar al Señor con los labios', y c) cambios de expresiones no figuradas por otras figuradas, como 'confiar' por 'apoyarse' ${ }^{15}$.

Newmark plantea seis opciones. En referencias extraídas de Valero-Garcés, de acuerdo con la clasificación realizada por Newmark, los métodos son: 1) reproducción de la misma imagen en la lengua meta; 2) traducción de la imagen del texto fuente por otra estándar en la lengua meta que no contraste con su cultura; 3) traducción de la metáfora por un símil al que se le adiciona una ampliación del significado; 4) traducción de la metáfora a través de una explicación de su significado; 5) eliminación de la metáfora, cuando ésta sea redundante e innecesaria y cuando no constituye un rasgo estilístico del autor; finalmente 6) retención de la misma metáfora acompañada de una explicación de su significado ${ }^{16}$.

Hay, pues, discrepancia entre los autores. Mientras que Nida y Taber están a favor de agregar metáforas donde no las hay en el texto de partida, Newmark elimina las existentes cuando éstas sean accesorias y superfluas. No nos queda claro, tampoco, cuando nos dice Newmark que se puede reproducir la misma imagen en la lengua de llegada; o cuando propone transferir la imagen del texto original (TO) por otra estándar en la lengua meta que no desentone con su cultura.

15 Eugene Nida y Charles R. Taber, La traducción: teoría y práctica (Leiden: E.J. Brill, 1982) 146.

16 Carmen Valero-Garcés, Languages in Contact: An Introductory Textbook on Translation (Maryland, EUA: University Press of America, 1995) 221-222. 
¿Quiere decir eso que uno de los métodos es la traducción literal de la metáfora? Y al plantear que traslademos una imagen intrínseca de la cultura del TO a la cultura meta y que no contraste con ella, iespera equivalencia semántica, elocusoria, formal, o acaso debemos sacrificar la metáfora original en función de encontrar otra propia de la cultura de llegada? ¿Cuál se debe aplicar para la supresión de una metáfora? ¿Acaso no le proporciona esta alternativa la libertad de decidir sobre qué traducir y qué no traducir, qué conservar y qué desechar? Burton Raffel siente las mismas inquietudes y en torno a la traducción de las metáforas presentes en el Quijote, se pregunta «Is this metaphor really worth all the trouble?» ¿Acaso? se cuestiona, ¿no sería más fácil omitirlas y no tener que luchar con ellas?

The answer, it seems to me, is the key to really translating Cervantes' novel, and though the answer given by earlier translators has been a consistent no, it is not worth it at all, we have other fish to fry, I would insist that if Cervantes thought the metaphor important enough to put into his masterpiece, the translator had damned well better find it of equal importance... ${ }^{17}$

Si se considera que no todas las estrategias formuladas por los teóricos son adecuadas, y que la clasificación de los tipos de metáfora es un tema en el que pocos concuerdan, partiremos con el análisis de las elecciones de Henry desde un punto de vista práctico y funcional, limitándonos a observar qué se hizo, a la vez que planteamos una evaluación de la propuesta de la traductora. Se han seleccionado algunos ejemplos representativos de las técnicas que se comentarán.

17 Burton Raffel, The Art of Translating Prose (University Park, PA: Penn State University Press, 1994) 133. 


\section{Cuadro 1. Omisión de la metáfora}

\begin{tabular}{|l|l|}
\hline \multicolumn{1}{|c|}{ Texto fuente } & \multicolumn{1}{c|}{ Texto meta } \\
\hline $\begin{array}{l}\text { ¿Quién había metido a sus parientes a } \\
\text { ser tan jueces y tan verdugos...? (22) }\end{array}$ & $\begin{array}{l}\text { Who had caused her family to be such } \\
\text { cruel judges of her...? (48) }\end{array}$ \\
\hline al sazonón de nor Pascual (58) & Señor Pascual (56) \\
\hline
\end{tabular}

En los ejemplos del cuadro 1, se observa reducción léxica en el texto terminal (TT), en el que la imagen de parientes verdugos ha sido sustituida por un adjetivo que equipara la frase original; no obstante, se le resta valor a la imagen del texto fuente. En el segundo caso, la carga semántica del adjetivo sazonón es omitido del todo. Se pierde la idea original de hombre maduro y conservado que refuerza y rescata la caracterización del personaje de Pascual que el narrador ya había adelantado al principio de la historia. Los casos en que se eliminó la metáfora son solamente los dos anteriores, y se puede notar que esa ausencia no altera en un grado significativo el valor total de la narración.

\section{Cuadro 2. Traducción literal de la metáfora}

\begin{tabular}{|l|l|}
\hline \multicolumn{1}{|c|}{ Texto fuente } & \multicolumn{1}{c|}{ Texto meta } \\
\hline $\begin{array}{l}\text { le tuvieran el maldito cafetalillo todo } \\
\text { dado a Satanás a punta de bolazos (13) }\end{array}$ & $\begin{array}{l}\text { falling entirely into Satan's hands by } \\
\text { dint of swipes with a ball (19) }\end{array}$ \\
\hline $\begin{array}{l}\text { lo negro se había tornado blanco y lotur- } \\
\text { bio saltado a claro por una simple... (13) }\end{array}$ & $\begin{array}{l}\text { black became white and what was dim } \\
\text { became clear by means of a simple...(21) }\end{array}$ \\
\hline $\begin{array}{l}\text { hacer un nudo y en seguida no saber } \\
\text { cómo desenredarlo! (43) }\end{array}$ & $\begin{array}{l}\text { tie a knot and then not know how to } \\
\text { untie it! (45) }\end{array}$ \\
\hline la soltó [la lengua] por el camino (43) & he loosened his tongue (45) \\
\hline no trepa sino a ser cristiano a secas (44) & $\begin{array}{l}\text { doesn't rise to be more than an unre- } \\
\text { markable fellow (46) }\end{array}$ \\
\hline $\begin{array}{l}\text { se pasó por la cara como nueve caras } \\
\text { distintas (49) }\end{array}$ & $\begin{array}{l}\text { she let pass over her face like nine } \\
\text { different expressions (49) }\end{array}$ \\
\hline allá por donde nacen los relámpagos (57) & $\begin{array}{l}\text { to the place where lightning comes } \\
\text { from (55) }\end{array}$ \\
\hline $\begin{array}{l}\text { tan de barro suave con el que podían } \\
\text { hacerse desde ollas hasta comales (57) }\end{array}$ & $\begin{array}{l}\text { of such soft clay as pots and griddles } \\
\text { can be made of (56) }\end{array}$ \\
\hline
\end{tabular}


El recurso ejemplificado en el cuadro 2 permite la traducción palabra por palabra, siempre que la imagen creada en la lengua de llegada no active estructuras, frames en $\operatorname{Katan}^{18}$, no contenidas en la metáfora original. El traductor, como mediador cultural en textos caracterizados por una singular riqueza idiosincrática, ha de realizar un proceso de decodificación para discernir esos espacios donde el trasvase literal no drene el texto del espíritu que el escritor depositó en él. Además, se debe advertir qué factor priva en las palabras que intervienen en la figura, su valor denotativo o sus connotaciones, y hasta qué punto esos valores los comparten ambas culturas; así, la decisión de traducir literalmente no es arbitraria, al considerar los aspectos sociolingüísticos y pragmáticos que intervienen en la creación de un texto cultural ${ }^{19}$.

\section{Cuadro 3. Neutralización o naturalización de la metáfora}

\begin{tabular}{|l|l|}
\hline \multicolumn{1}{|c|}{ Texto fuente } & \multicolumn{1}{c|}{ Texto meta } \\
\hline un negro pecado (13) & a grave sin (20) \\
\hline se cría lleno de grietas y endebleces (44) & grows up full of faults and weaknesses (45) \\
\hline $\begin{array}{l}\text { porque trompicó la echaron al barreal a que } \\
\text { se ahogara (45) }\end{array}$ & $\begin{array}{l}\text { when she got into trouble, and they cast } \\
\text { her out into the swamp to drown (47) }\end{array}$ \\
\hline $\begin{array}{l}\text { algún adiós y algún buenos días le dejara } \\
\text { caer (45) }\end{array}$ & $\begin{array}{l}\text { there were some who spoke to her, saying } \\
\text { 'adiós' and 'buenos días' (47) }\end{array}$ \\
\hline el turno dio muy buena cosecha (47) & the show brought in a lot of money (48) \\
\hline $\begin{array}{l}\text { vino de dundo a meter sus patotas para que } \\
\text { se las amarrara (47) }\end{array}$ & $\begin{array}{l}\text { came and put himself at the mercy of } \\
\text { Auristela (48) }\end{array}$ \\
\hline $\begin{array}{l}\text { se fue chapaleando dudas en un gran charco } \\
\text { de incertidumbre (48) }\end{array}$ & $\begin{array}{l}\text { went off amid doubts and much uncertain- } \\
\text { ty (48) }\end{array}$ \\
\hline $\begin{array}{l}\text { ya tenía alunados los lomos a punta de tanta } \\
\text { albarda (51) }\end{array}$ & $\begin{array}{l}\text { and was already suffering the effects of } \\
\text { such a hard life (51) }\end{array}$ \\
\hline [Auristela] no estaba mal sazonada (51) & was good-looking (51) \\
\hline enroscado en la cabeza (56) & she'd got me thinking about her (55) \\
\hline a contarme todo el tamal, ya sin hojas (60) & tell me the whole story, straight (58) \\
\hline
\end{tabular}

18 David Katan, Translating Cultures (Manchester, UK: St. Jerome, 1999).

19 Hurtado expone la dinámica del binomio lengua-cultura en el capítulo 4 «El traductor como mediador cultural». Catalina Jiménez Hurtado, La estructura del significado en el texto (Granada: Editorial COMARES, 2000). 
Nida (1964) y Vinay y Darbelnet (1958/1960), citados por Samaniego, indican que en la mayoría de las ocasiones la traducción literal de la metáfora no es viable y hay que sustituir el término metafórico por uno no metafórico ${ }^{20}$. Esta aseveración es válida en aquellos casos en que el significado literal de los lexemas que componen la figura al ser combinados en la lengua meta no corresponden al sentido al que apunta el mensaje original. A veces se opta por una traducción literal forzada y el resultado es confuso para el lector meta. Estamos de acuerdo en que el traductor de un texto cultural debe dotar a su versión del efecto de extrañeza del original, que se está leyendo una traducción, pero sin perder de vista que ante todo, siendo un mediador cultural, el traductor debe crear ese puente que le permita a sus lectores entrar en contacto con las ideas del autor, aunque el valor estético no el semántico, haya tenido que modificarse. Sobresale de entre los ejemplos planteados el caso en que la frase al inglés conserva ciertos elementos del español, específicamente los saludos (there were some who spoketo her, saying 'adiós 'and 'buenos dias'). Es clara la intención de la traductora por transmitir el exotismo del que comentábamos y, a pesar de que se pierde la metáfora, el elemento cultural le recuerda al lector que la situación y los personajes provienen de otras latitudes, con otras costumbres y otras circunstancias. 


\section{Cuadro 4. Simplificación léxica o sintáctica con pérdida de la fuerza semántica o elocusoria}

\begin{tabular}{|l|l|}
\hline \multicolumn{1}{|c|}{ Texto fuente } & \multicolumn{1}{c|}{ Texto meta } \\
\hline $\begin{array}{l}\text { el viento se había quedado descansando en } \\
\text { el camino (7) }\end{array}$ & the wind had died down (15) \\
\hline $\begin{array}{l}\text { por dentro me hormigueaban dudas grandes } \\
\text { como toronjas (10) }\end{array}$ & huge doubts teemed within me (17) \\
\hline $\begin{array}{l}\text { por más que la conciencia se le hiciera una } \\
\text { enredazón de mecates (13) }\end{array}$ & $\begin{array}{l}\text { however mixed up his conscience became } \\
(20)\end{array}$ \\
\hline $\begin{array}{l}\text { con todo y lo espinado de su cáscara de } \\
\text { chayote sazón (46) }\end{array}$ & in spite of his prickly, surly nature (48) \\
\hline le había descascarado sus buenas tajadas (47) & had got a good rake-off (48) \\
\hline $\begin{array}{l}\text { le sonsacó así con cucharón grande buenas } \\
\text { rebanadas de las intimidades de su... (50) }\end{array}$ & $\begin{array}{l}\text { wheedled out of him many an intimate } \\
\text { detail about his life (50) }\end{array}$ \\
\hline resultando después, que ni un quelite (57) & he became still weaker after that (56) \\
\hline traer al terreno de las verdades a Eustaquio (58) & when she told him! (57) \\
\hline
\end{tabular}

En el cuadro 4 se observa que aunque se mantienen las metáforas, a excepción del penúltimo caso que bien pudo haberse clasificado dentro de la categoría anterior, lo evidente es el grado de simplificación sintáctica y léxica que en todas las instancias ha dado como resultado una disminución de la carga semántica y del poder elocusorio de la figura. No corresponde esta merma a la estrategia anterior, en que se analizó la neutralización o naturalización de la metáfora, lo que justifica el penúltimo ejemplo. Se rescata el esfuerzo por conservar la figura literaria y discrepamos con Dagut, quien opina lo siguiente:

Es totalmente imposible 'medir' de modo exacto la originalidad o el grado de invención que cada figura muestra en el idioma origen; por lo tanto, al no poder calcular de modo ni siquiera aproximado el efecto o impacto que la metáfora le ha supuesto al lector del Texto Origen, es imposible reproducir algo tan disperso en el idioma meta ${ }^{21}$.

Los fragmentos de ambos textos prueban un acercamiento entre las dos realidades lingüísticas, porque ambos sistemas culturales lo 
permiten y, a pesar de que se pierde parte de la carga semántica y elocusoria, la traductora ha capado «the frames of interpretation in the source language [y de crear] a similar set of interpretation frames to be accessed in the target reader's mind $»^{22}$.

\section{Cuadro 5. Metáfora equivalente en la cultura meta}

\begin{tabular}{|l|l|}
\hline \multicolumn{1}{|c|}{ Texto fuente } & \multicolumn{1}{c|}{ Texto meta } \\
\hline usted se trae abejón en el buche (7) & you've got a bee in your bonnet (15) \\
\hline $\begin{array}{l}\text { ah, peso, muchacho, el que me he lim- } \\
\text { piado de encima (9-10) }\end{array}$ & $\begin{array}{l}\text { Ah, the burden, my lad, which I've } \\
\text { taken off my shoulders (17) }\end{array}$ \\
\hline $\begin{array}{l}\text { cómo se las había mandado estirar la } \\
\text { pata ñor Evaristo (13) }\end{array}$ & $\begin{array}{l}\text { how Señor Evaristo had managed to } \\
\text { peg out (20) }\end{array}$ \\
\hline $\begin{array}{l}\text { comenzó a picarle la punta de la lengua } \\
\text { (43) }\end{array}$ & $\begin{array}{l}\text { he couldn't hold his tongue any longer } \\
\text { (45) }\end{array}$ \\
\hline $\begin{array}{l}\text { armarse de alguna mala fama y tam- } \\
\text { bién de alguna buena platilla (45) }\end{array}$ & $\begin{array}{l}\text { she got herself some bad reputation } \\
\text { and a good meal into the bargain (46) }\end{array}$ \\
\hline $\begin{array}{l}\text { pero de qué valía espantar piuses ahora } \\
\text { cuando todo el maíz ya había ido lejos } \\
\text { (52) }\end{array}$ & $\begin{array}{l}\text { But what was the use of bolting the } \\
\text { stable door when the horse had already } \\
\text { gone? (52) }\end{array}$ \\
\hline dejándonos a oscuras (57) & leave us in the dark (55) \\
\hline el mundo se le vino a bajo (59) & the world collapsed for my relative (57) \\
\hline
\end{tabular}

Este es el método ideal. No obstante, la experiencia enseña que la equivalencia uno a uno no es la más frecuente; de ahí haya que echar mano de otras técnicas. Vázquez Ayora explica que «teóricamente en la mente humana pueden surgir infinitas situaciones conceptuales muy complejas, aparentemente imposibles. El traductor debe verterlas haciéndolas lo más psicológicamente posibles» ${ }^{23}$ y lo más semántica y pragmáticamente cercanas. Insiste en que «el lenguaje figurado, como las expresiones de significación exocéntrica, rehúsan la traducción directa y requieren sus propias técnicas de transferencia a otras

22 Katan, 125.

23 Gerardo Vázquez Ayora, Introducción a la traductología (Georgetown: Georgetown University, 1977), 59. 
lenguas $»^{24} \mathrm{y}$ propone tres métodos para trasvasar una metáfora a otra lengua: mediante «(a) modulación de una metáfora transformándola en una expresión no metafórica, (b) modulación de una metáfora a un símil y (c) equivalencia de una metáfora con otra» ${ }^{25}$.

\section{Cuadro 6. Conversión a símil con o sin ampliación}

\begin{tabular}{|l|l|}
\hline \multicolumn{1}{|c|}{ Texto fuente } & \multicolumn{1}{c|}{ Texto meta } \\
\hline noches de gata de tejado (45) & $\begin{array}{l}\text { Like a cat she got used to nights on the } \\
\text { tiles (46) }\end{array}$ \\
\hline $\begin{array}{l}\text { esta clase de mujeres hechas a macha- } \\
\text { martillo, que tan se acuestan diablos } \\
\text { como amanecen ángeles (50) }\end{array}$ & $\begin{array}{l}\text { this type of absolutely determined } \\
\text { women who go to bed like devils and } \\
\text { get up like angels (51) }\end{array}$ \\
\hline
\end{tabular}

Al igual que los casos de omisión, las metáforas sustituidas por símiles se idntificaron en dos ocasiones (ver cuadro 6). Esta operación es defendida por Newmark ${ }^{26}$ y Larson ${ }^{27}$, sobre la cual Esteban Torre se pronuncia:

En algunas lenguas, puede ser conveniente cambiar la metáfora por un símil... a fin de hacer el TLT [texto en la lengua terminal] más comprensible. Cuando en la traducción se hace mención explícita del rasgo semántico común entre el término metaforizado y el término metafórico, existen escasas posibilidades de una interpretación errónea ${ }^{28}$.

26 Peter Newmark, Approaches to Translation (Oxford: Pergamon Press, 1982) 90.

27 En Teoría de la traducción literaria, de Esteban Torre, pag. 147 y La traducción de la metáfora de Eva Samaniego Fernández, pag. 110.

28 Torre, 147. 


\section{Cuadro 7. Casos varios: falsas interpretaciones, cambios de sentido y cambios de registro}

\begin{tabular}{|l|l|}
\hline \multicolumn{1}{|c|}{ Texto fuente } & \multicolumn{1}{c|}{ Texto meta } \\
\hline $\begin{array}{l}\text { hasta piedras había tenido que digerir } \\
\text { en su vida (51) }\end{array}$ & she had had to live very frugally (51) \\
\hline babear de contento (8) & was glowing with pleasure (56) \\
\hline $\begin{array}{l}\text { y los cuyeos salpimentaban aquí y allá } \\
\text { el ancho silencio nocturno (8) }\end{array}$ & $\begin{array}{l}\text { and the guinea-pigs here and there } \\
\text { broke the overall silence of the night } \\
(16)\end{array}$ \\
\hline $\begin{array}{l}\text { como si los latines le hubieran sabido } \\
\text { a rancio (9) }\end{array}$ & $\begin{array}{l}\text { And as if the Latin tags had known } \\
\text { they were ancient (17) }\end{array}$ \\
\hline $\begin{array}{l}\text { debió de andar muy apurada [el alma] } \\
\text { en lo de San Pedro y sus tremendas } \\
\text { llaves (13) }\end{array}$ & $\begin{array}{l}\text { must have to go at top speed to Saint } \\
\text { Peter and his awesome keys (21) }\end{array}$ \\
\hline $\begin{array}{l}\text { La tal había de muy ternerilla quedado } \\
\text { aumentada (43) }\end{array}$ & $\begin{array}{l}\text { This woman had grown very stout, } \\
\text { made pregnant by some unknown } \\
\text { man (45) }\end{array}$ \\
\hline $\begin{array}{l}\text { ¿Acaso mi comadre servía para apun- } \\
\text { tarse a cosa de perder? (47) }\end{array}$ & $\begin{array}{l}\text { Perhaps she acted to join a lost } \\
\text { cause? (48) }\end{array}$ \\
\hline $\begin{array}{l}\text { p. } 51 \text { : había sacado la cabeza de entre } \\
\text { las piedras (47) }\end{array}$ & $\begin{array}{l}\text { had kept her head above water until } \\
\text { now (51) }\end{array}$ \\
\hline puso abajo (57) & he sat down again (55) \\
\hline la cosa estaba a punto de jalea (58) & $\begin{array}{l}\text { there was proof that they were } \\
\text { madly in love (57) }\end{array}$ \\
\hline no me quedo con basura en el ojo (60) & And as there's no mud in my eye (58) \\
\hline
\end{tabular}

En el cuadro 7 hay dos casos en los que se ha percibido un cambio de registro, de un tono muy coloquial y popular a otro más formal y neutral. Tanto estos giros como los incluidos en adelante revelan un desconocimiento del sentido del mensaje original, así como de algunas construcciones gramaticales que han impedido proponer un equivalente adecuado en lengua inglesa. En el tercer ejemplo, 'cuyeo' se refiere a un ave que emite un sonido particular que se aprovecha por onomatopeya, ha sido traducido por guinea-pigs, probablemente porque en las acepciones del diccionario aparecen $c u y^{29}$ y cuye o cui

29 María Moliner, Diccionario del uso del español (Madrid: Gredos, 1987) 407. 
(o cuy), pluralizado en cuyes o cuis y cuise $^{30}$, y en que en todos los casos nombra a un conejillo de Indias. Se entiende el error, pues este es un nombre propio conocido solamente en la escena rural y que poco transciende más allá de ese espacio reducido menos aún fuera de nuestras fronteras.

Los ejemplos 6 y 9 confirman el tropiezo léxico de la traductora, ya que la realidad a la que hacen referencia las frases 'muy ternerilla' y 'puso abajo'es respectivamente 'muy joven'y 'accedió'. El penúltimo ejemplo nos habla de una situación que está en su mejor momento, algo así como 'a punto de caramelo', con la excepción que el autor prefiere acercarlo a la realidad costarricense y emplea 'jalea' en lugar del otro dulce. El último caso dice que el personaje no es de los que se quedan con la duda o la inquietud. Sin embargo, Henry propone una frase que no funciona, pues no consigue transmitir la idea del original. Sobre las discrepancias en el plano léxico opina Ivonne Lindqvist:

If source and target text are parts of two widely different cultures, it is very likely that the lexicon of the two languages will differ essentially. Nevertheless, even if the cultures are fairly close they will by no means share all concepts in the lexicon. There is no one-to-one relationship of lexical concepts in one language to another ${ }^{31}$.

\section{Conclusiones: En torno a la traducción de las metáforas en His- torias de Tata Mundo}

Esteban Torre es enfático al aclarar lo siguiente:

Metáfora, sinécdoque y metonimia no significan en griego más que 'traslado', 'deslizamiento', 'cambio de nombre'... No tiene así pues, nada de extraño que algún autor moderno (Newmark, 1992:147)

30 Real Academia Española, Diccionario de la Lengua Española, $21^{a}$ ed. (Madrid: Espasa Calpe, 1992), 622, 631.

31 Peter Jansen, ed. The Manipulation of Discourse, (Leuven: CETRA, 1992-1993) 94. 
haya podido, con toda razón, escribir: 'Entiendo por metáfora cualquier expresión figurada ${ }^{32}$.

Eva Samaniego realiza un estudio exhaustivo de la metáfora como fenómeno interlingüístico, en el que compara la posición de un número significativo de lingüistas y autores literarios en cuanto a la naturaleza y características de la figura y su traducibilidad. La autora se enfrenta a opiniones contrastantes y algunas poco alentadoras que ratifican la imposibilidad de trasvasar la metáfora de una lengua a otra. No obstante, resume «la postura conciliadora está representada por Snell-Hornby (1988), que opina que ni la metáfora es intraducible, ni es totalmente traducible: la posibilidad de trasvase depende del texto en que se halle inserta y de factores $a d h o c »^{33}$.

Grosso modo, la labor de Joan Henry en The Stories of Tata Mundo supera la prueba de la traducibilidad, debido a que el texto de salida es una obra que condensa en pocas páginas una variedad de elementos culturales muy arraigados al personaje campesino de mediados de siglo xx y del que poco o nada conoce la traductora, excepto quizás por su contacto con el mismo autor y otras obras similares. Newmark plantea la eliminación como una operación válida cuando la figura no cumple una función estratégica y sólo después de que el traductor ha medido la carga semántica y tiene la certeza de no restarle valor al contexto particular en que se inserta la metáfora $y$ a la obra en general ${ }^{34}$.

Hay un empleo frecuente de la naturalización o paráfrasis de las metáforas originales; sin embargo, el recurso se justifica debido al grado de dificultad del texto original. En casos en que el mensaje y el contenido debe prevalecer sobre la forma, la neutralización de algunas frases figuradas de uso idiosincrático es tolerable y hasta aceptable. Nida insiste en mantener el sentido al trasvasar un texto

34 Approaches to Translation, 91. 
de una lengua a otra; opina que la forma es secundaria. Con algunas excepciones, tal es el caso de la poesía:

En cada lengua las reglas para relacionar el contenido con la forma son muy complejas, arbitrarias y variables... Tanto mejor si, por coincidencia, se puede transmitir el mismo contenido en la lengua receptora con una forma muy parecida a la del original; mantenemos la forma cuando podemos, pero muy a menudo ésta tiene que ser modificada precisamente para mantener el contenido. Un esfuerzo excesivo por conservar la forma lleva inevitablemente a una grave pérdida o distorsión del mensaje ${ }^{35}$.

La traducción literal es otra operación al alcance del traductor, cuando una metáfora equivalente no existe en la lengua de llegada y la unión de los lexemas provoque confusión en el lector meta, a menos sea la intención del original. Samaniego cita a Klopfer (1967) y Reiss (1971), quienes sostienen que «esta figura no es un problema para la traducción puesto que siempre existe la posibilidad de traducir 'palabra por palabra'» ${ }^{36}$.

A diferencia de Dagut, quien rechaza la idea «que una metáfora pueda ser reproducida mediante creación, es decir, mediante un equivalente en la Lengua Meta» ${ }^{37}$, compartimos el que en instancias específicas en que el texto presenta un número considerable de metáforas novedosas u originales, como en la obra Historias de Tata Mundo, el traductor pueda recrearlas en su lengua. A fin de cuentas, el proceso de decodificación y recodificación o deconstrucción y reconstrucción le permite convertirse en el canal que transporta la realidad del texto fuente al lector meta, y siendo el traductor consciente de este poder, afronta la responsabilidad de mantenerse ante todo leal a la intención del autor y su obra, y si por añadidura puede contribuir a enriquecer el entorno lingüístico y literario de su cultura todavía mejor. Biguenet y

$\overline{35}$ Nida y Taber, 144.

36 Samaniego, 84.

37 Samaniego, 82. 
Schulte opinan que ante la traducción de la metáfora y la disyuntiva que genera, el traductor no debe desistir, pues está a posibilidad de hacer a un lado la traducción estándar y de buscar «something new that will both give the meaning of what the author wants to say and make it quite clear that something new has been added to both languages $\gg^{38}$.

La traducción literariaes un complejo procedimiento: «a complex procedure, inevitably involving two languages and two literary traditions, that is, two sets of norm-systems $»^{39} ; \mathrm{y}$ cada obra ocupa el lugar exacto en el polisistema literario de salida como texto original y en el de llegada como una representación de un texto ya existente, el cual por una u otra razón ha sido favorecido con la renovación y divulgación que se obtiene a partir de su traducción a otro idioma. Dado que en el marco de la región latinoamericana, la historia costarricense no registra conflictos épicos o situaciones particularmente extraordinarias, la producción literaria local ha padecido cierta desatención por parte del lector de lengua extranjera. Por ello, los esfuerzos de traductores y editoriales que apoyen y difundan la labor de letras nacionales no deben dejarse de lado. 